\title{
Changes of property and morphology of cationic corn starches
}

\author{
Wan-Yuan Kuo, Hsi-Mei Lai * \\ Department of Agricultural Chemistry, National Taiwan University, Taipei, Taiwan
}

Received 5 October 2006; received in revised form 26 December 2006; accepted 13 January 2007

Available online 26 January 2007

\begin{abstract}
Cationic starches with the degree of substitution (DS) ranging from 0.0075 to 0.1215 were prepared from the reaction of native corn starch with cationizing agent (3-chloro-2-hydroxypropyl)trimethylammonium chloride (CHPTAC) under the molar ratios of CHPTAC/ $\mathrm{AGU}$ (anhydroglucose unit) $=1$ and 1.71 in aqueous suspension at $40{ }^{\circ} \mathrm{C}$ for different reaction times $(2,5$ and $24 \mathrm{~h})$. Pasting and thermal properties of the cationic starches were evaluated by using a rapid-visco-analyser and differential scanning calorimetry, respectively. Morphological changes of the cationic starches were investigated by using scanning electron microscopy, confocal laser scanning microscopy, polarized optical microscopy, bright field microscopy, and wide angle X-ray diffractometry. The changes in the pasting and thermal properties, correlated well with the increase of DS, together with the characterizations of granular morphology of the cationic starches, pointed out the gradual reduction of the structural integrity of cationic starches with the rising in DS up to 0.0431, while evident physicochemical and morphological changes took place when DS was raised to 0.1215 .
\end{abstract}

(c) 2007 Elsevier Ltd. All rights reserved.

Keywords: Cationic starch; Degree of substitution; Pasting property; Thermal property; Granular morphology; Confocal laser scanning microscopy $(\mathrm{CLSM})$

\section{Introduction}

Cationic starch is an important starch derivative used in numerous applications. It is usually used in paper manufacturing to improve mechanical strength, retention of fines, dyes and fillers, faster drainage, running of paper machine, and to decrease biological oxygen demand of paper mill effluent (Jensen \& Winters, 1986; Nachtergaele, 1989; Wurzburg, 1986). Cationic starch is also used as a flocculant for water treatment (Krentz et al., 2006), or additive in textile, cosmetic products (Heinze, Haack, \& Rensing, 2004). Recently, some researchers have made cationic starch dynamic coating additive for protein analysis in capillary electrophoresis (Sakai-Kato et al., 2006). Cationic starch is produced from the reaction of starch with reagents containing amino, imino, ammonium, sulphonium or phosphonium groups (Solarek, 1986). Different

\footnotetext{
* Corresponding author. Tel.: +8862 33664816; fax: +886223633123.

E-mail address: hmlai@ntu.edu.tw (H.-M. Lai).
}

methods have been described to make cationic starch. In the extrusion process, reagent is added to the dry starch during extrusion (Gimmler \& Meuser, 1994; Valle, Colonna, \& Tayeb, 1991); in a semi-dry method, reagent is sprayed onto the starch then the mixtures are exposed to heat treatment (Hellwig, Bischoff, \& Rubo, 1992). Both extrusion and semi-dry method minimize the amount of waste water. However, salts, residual reagents or reagent by-products may be present in the final products (Radosta et al., 2004). Another method to make cationic starch is the wet method, in which reactions are processed homogeneously in DMSO or heterogeneously in an aqueous suspension under alkaline conditions (Heinze et al., 2004) to enhance diffusion of the cationizing agent into starch granules and to catalyze the etherification reaction. Controlling of temperature and addition of $\mathrm{Na}_{2} \mathrm{SO}_{4}$ are sometimes used to avoid starch gelatinization due to high $\mathrm{pH}$ and high temperature of the reaction, but add to the need for extensive washing and effluent treatment of the spent reaction medium and washings. Cationization of starch in an aqueous alcoholic-alkaline system avoids gelatinization of starch 
granules and eliminates the requirement for swelling-inhibiting salts (Kweon, Hanover, Sosulski, \& Han, 1997), but still generates organic solvent wastes. Besides, inhibition of granular swelling might lower the reaction efficiency of modified starches (Huber \& BeMiller, 2003). The development of new methods for starch cationization is still in progress. Siemion, Kapusniak, and Koziol (2005) treated the mixtures of starch and semicarbazide hydrochloride with either conventional or microwave heating and obtained a product that contained cationic groups.

Physicochemical properties as well as granular morphology of starch are altered after cationization. Generally, lower pasting temperature, higher peak viscosity, and various changes in setback were observed in the rapid-viscoanalysis (RVA) of different kinds of starch (Kweon et al., 1997; Siau, Karim, Norziah, \& Wan Rosli, 2004). Smaller gelatinization enthalpy and lower transition temperature of cationic starches compared to native starches were reported from the analysis using differential scanning calorimetry (DSC) (Radosta et al., 2004; Yook, Sosulski, \& Bhirud, 1994). Under the examination of scanning electron microscopy (SEM), cationic potato starches derived from water suspension were slightly swollen, while those derived from solvent or dry cationization were merely unchanged in their granular forms, except for the presence of some fine particles (Vihervaara, Bruun, Backman, \& Paakkanen, 1990). The cationic substitution by aqueous methods is believed to occur mainly at the amorphous region, as evidenced by the unchanged X-ray diffraction patterns (Aggarwal \& Dollimore, 1998; Yook et al., 1994) and remained birefringence under polarized light microscopy (Radosta et al., 2004). To the best of our understanding, most researches about cationic starches process cationization in alcohols or added with swelling-inhibiting salts to prevent the starches from complete gelatinization; while few researches have worked on the preparation of salts-free aqueous cationization of granular starches. Furthermore, the use of confocal laser scanning microscopy (CLSM) to investigate the granular characteristics of cationic starch has not been reported. Since the structure of starch is related to its functional properties, which affect its applications, it is important to understand the relationships between physicochemical properties and granular morphology of cationic starch. Therefore, the purpose of this paper is to study the effects of cationization on the physicochemical properties and granular structures of cationic starches derived from water phase cationization, and to discuss the relationships between the physicochemical properties and granular structures of the cationic starches.

\section{Experimental}

\subsection{Materials}

Native corn starch was purchased from Gu-Tong Food Industry Ltd. (Chiayi, Taiwan). The cationizing agent CHPTAC ((3-chloro-2-hydroxypropyl)trimethylammoni- um chloride) was provided by Aldrich Chemical Co. (Milwaukee, WI, USA). $\mathrm{NaOH}$, ethanol and $\mathrm{HCl}$ were purchased from Nacalai Tesque, Inc. (Kyodo, Japan), Taiwan Sugar Corporation (Tainan, Taiwan), and SigmaAldrich Co. (Taufkirchen, Germany), respectively.

\subsection{Preparation of cationic starches}

Native corn starch (90 g, on dry basis) was suspended in $100 \mathrm{~mL}$ of $1 \mathrm{mM} \mathrm{NaOH}$ and mixed with $150.8 \mathrm{~g}$ or $297.1 \mathrm{~g}$ CHPTAC, which had been adjusted to $\mathrm{pH} 12$ with $5 \mathrm{~N}$ $\mathrm{NaOH}$. The mixture solution was adjusted to $\mathrm{pH} 11$ with $2 \mathrm{~N} \mathrm{NaOH}$, then diluted with $1 \mathrm{mM} \mathrm{NaOH}$ to $600 \mathrm{~mL}$. The reaction was carried out at $40^{\circ} \mathrm{C}$ with continuous stirring $(200 \mathrm{rpm})$. One-third amount by weight of the reaction mixture was taken away at 2, 5 and $24 \mathrm{~h}$ each time. After diluting the reaction mixture at specific reaction time with an equal volume of $95 \%$ ethanol, the reaction mixture was centrifuged at $10,000 \mathrm{~g}$ for $10 \mathrm{~min}$ and the precipitate was collected. The collected precipitate was resuspended in $63 \%$ ethanol $(300 \mathrm{~mL})$ and neutralized with $4.8 \mathrm{~N} \mathrm{HCl}$. The cationic starch was washed with $63 \%$ ethanol several times till free of chlorides in washing solution. The obtained cationic starch was dried at $40^{\circ} \mathrm{C}$ overnight. The Kjeldahl method described by AACC (2000) was used to determine the nitrogen content of the cationic starches. Degree of substitution (DS) of each sample was calculated using the equation below (Heinze et al., 2004) and the determinations of DS were run in triplicates for each sample.

$$
\mathrm{DS}=\frac{(162.15 \times \% \text { nitrogen })}{1401-(154.64 \times \% \text { nitrogen })}
$$

\subsection{Pasting properties}

The rapid-visco-analyser (RVA4, Newport Scientific Pty, Ltd., Warriewood, Australia) was used to determine the pasting properties of the cationic starches. Cationic starch suspension $\left(8 \%(\mathrm{w} / \mathrm{w})\right.$, on dry basis) was held at $35^{\circ} \mathrm{C}$ for $1 \mathrm{~min}$, heated to $95^{\circ} \mathrm{C}$ in $20 \mathrm{~min}$, held at $95^{\circ} \mathrm{C}$ for $10 \mathrm{~min}$, cooled to $50^{\circ} \mathrm{C}$ in $20 \mathrm{~min}$, then held at $50^{\circ} \mathrm{C}$ for $2 \mathrm{~min}$. The paddle speed was $960 \mathrm{rpm}$ for the first $10 \mathrm{~s}$, and maintained at $160 \mathrm{rpm}$ to the end of the test. For $24 \mathrm{~h}$-cationized starch, $2.54 \%(\mathrm{w} / \mathrm{w})$ starch suspension was used and the paddle speed was set at $160 \mathrm{rpm}$ from the beginning to the end of the test. Pasting temperature (PT), peak temperature $\left(P_{\mathrm{t}}\right)$, peak viscosity $(\mathrm{PV})$, hot paste viscosity $(\mathrm{HV})$, breakdown $(\mathrm{BD}=\mathrm{PV}-\mathrm{HV})$, final viscosity $(\mathrm{FV})$, and setback $(\mathrm{SB}=\mathrm{FV}-\mathrm{HV})$ were obtained from the measurements. Pasting properties of samples were run in triplicates and the coefficient of variance $(\mathrm{CV})$ of values derived from the pasting curve was between $0.6 \%$ and $10.1 \%$.

\subsection{Thermal properties}

Thermal characteristics of the cationic starches were studied using a modulated differential scanning calorimetry 
(MDSC 2910, TA Instruments, New Castle, DE, USA). A $10 \mathrm{mg}$ mixture containing $25 \%(\mathrm{w} / \mathrm{w})$ cationic starch and $75 \% \mathrm{dH}_{2} \mathrm{O}$ was weighed into an aluminum pan, sealed and equilibrated at room temperature for $6 \mathrm{~h}$. The sample was heated in an MDSC from 10 to $120^{\circ} \mathrm{C}$ at a heating rate of $5^{\circ} \mathrm{C} / \mathrm{min}$, an amplification of $\pm 1^{\circ} \mathrm{C}$, and a period of $40 \mathrm{~s}$, with an empty pan as a reference. The results were calculated with TA Instruments analyzing software. The transition temperatures including onset $\left(T_{\mathrm{o}}\right)$, peak $\left(T_{\mathrm{p}}\right)$ and complete $\left(T_{\mathrm{c}}\right)$ temperatures were recorded from a plot of heat flow vs temperature. The width (WD) and the gelatinization enthalpy $(\Delta \mathrm{H})$ were derived from the range of the transition temperatures and the conversion from the area under the curve to Joules referred to the indium standard, respectively. MDSC measurements were run in triplicates and the $\mathrm{CV}$ of the measurements was between $0.0 \%$ and $14.8 \%$.

\subsection{Scanning electron microscopy}

Granular shapes and the surface structures of the cationic starches were observed by using a scanning electron microscopy (SEM) (Hitachi S-2400, Tokyo, Japan). The sample was mounted on a circular aluminum stub with a double sticky tape, coated with gold by using an ion sputter (Hitachi E101, Tokyo, Japan) and examined by SEM at an accelerating potential difference of $20 \mathrm{kV}$.

\subsection{Confocal laser scanning microscopy}

Granular morphology of the cationic starches was observed by a confocal laser scanning microscopy (CLSM) (Leica TCS SP2 Confocal Spectral Microscope, Wetzlar, Germany) equipped with an argon laser. Starch granules were stained with aminofluorophore 8-amino-1,3,6-pyrenetrisulfonic acid (APTS, Molecular probes, Eugene, OR, USA) with the method modified from Chung and Lai (2006). Methanol was used as the solvent in the following dying steps: $3 \mathrm{mg}$ cationic starch was mixed with $15 \mu \mathrm{L}$ of $0.8 \mathrm{mM}$ APTS and $3 \mu \mathrm{L}$ of $1 \mathrm{M}$ sodium cyanoborohydride (Fluke, Milwaukee, WI, USA), and reacted at $30{ }^{\circ} \mathrm{C}$ for $15 \mathrm{~h}$. The mixture was then washed with $1 \mathrm{~mL}$ methanol for five times and the precipitate was resuspended in $10 \mu \mathrm{L}$ of $50 \%$ glycerol-methanol solution. An agar solution was prepared by boiling a methanol solution containing $2 \%$ agar and $85 \%$ glycerol for $5 \mathrm{~min}$ and cooled to $40^{\circ} \mathrm{C}$. The starch suspension $(5.5 \mu \mathrm{L})$ and the agar solution $(20 \mu \mathrm{L})$ were mixed. The mixture $(5.5 \mu \mathrm{L})$ was dropped onto the slide, covered with a cover glass, and observed by Leica TCS SP2 (Wetzlar, Germany) with the objective lens of $100 \times$ Plan apo/1.4 oil. The excitation wavelength was $488 \mathrm{~nm}$ ( $20 \%$ capacity) and the light was detected at intervals from 500 to $535 \mathrm{~nm}$. The format of image was $30 \times 30 \mu^{2}$ and $512 \times 512$ pixels. Each line was scanned eight times and averaged to reduce noise during image acquisition.

\subsection{Polarized optical microscopy and bright field microscopy}

Starch powder was spread onto a microscope slide, dropped with methanol, and covered with a cover glass spread with petroleum jelly at the periphery. The specimens were observed by a light microscope (Nikon ECLIPSE E600W POL, Tokyo, Japan) equipped with a cross-polarizer and a combined digital camera (Nikon COOLPI $\times 5400$, Tokyo, Japan). For iodine-stained starch, starch was suspended in methanol, dispersed onto a microscope slide, and dyed with one drop of iodine solution containing $1 \mathrm{mg} \mathrm{I}_{2}$ and $2.5 \mathrm{mg} \mathrm{KI}$ per milliliter of dye solution. A photo of the calibration slide at the same magnification was taken for size determination.

\subsection{Wide angle X-ray diffractometry}

X-ray diffraction patterns of native and the cationic starches were obtained using an X-ray diffractometer (Geigerflex, Regaku, Japan) operating at $15 \mathrm{~mA}, 35 \mathrm{kV}$ and at the $\mathrm{CuK}_{\alpha}$ wavelength $(0.1543 \mathrm{~nm})$. Samples equilibrated above saturated $\mathrm{BaCl}_{2}$ aqueous solution were pressed into a sample holder and scanned for $2 \theta$ from 3 to $40^{\circ}$ at a rate of $2^{\circ} / \mathrm{min}$ and an incremental interval of $0.1^{\circ}(2 \theta)$. The $\mathrm{X}$-ray diffractometry of each sample was run in triplicates.

\section{Results and discussion}

\subsection{Changes in physicochemical properties of cationic starches}

DS of the cationic starches prepared in this study fell between 0.0075 and 0.1215 , and increased with increasing in the amount of cationizing agent and the reaction time. This result corresponded with previous researches (Radosta et al., 2004; Siau et al., 2004). The pasting and thermal properties of the cationic starches with different DS were summarized in Table 1.

\subsubsection{Pasting properties}

Cationization significantly changed the pasting properties of all of the cationic starches (Table 1). Due to extremely high viscosity in cold water, cationic starches reacted for $24 \mathrm{~h}$ (CS1-24h and CS1.7-24h) had the concentration reduced from $8 \%$ to $2.54 \%$ for RVA measurements, and the discussion about their results were independent from other samples.

The cationic starches with DS $\leqq 0.0431$ showed strong correlations (Table 2) between DS and most of the pasting properties; i.e. the positive correlations of $\mathrm{PV}$ and $\mathrm{BD}$ but negative correlations of PT and $P_{\mathrm{t}}$ with DS. The cationic groups introduced onto the starch molecules repulsed each other due to both steric hindrance and Columbic repulsion and resulted in the inhibition of the inter-chain associations. As a result, a weaker granular structure of cationic starches permitted faster and greater water uptake compared to the native starch. Consequently, the low PT, $P_{\mathrm{t}}$ 


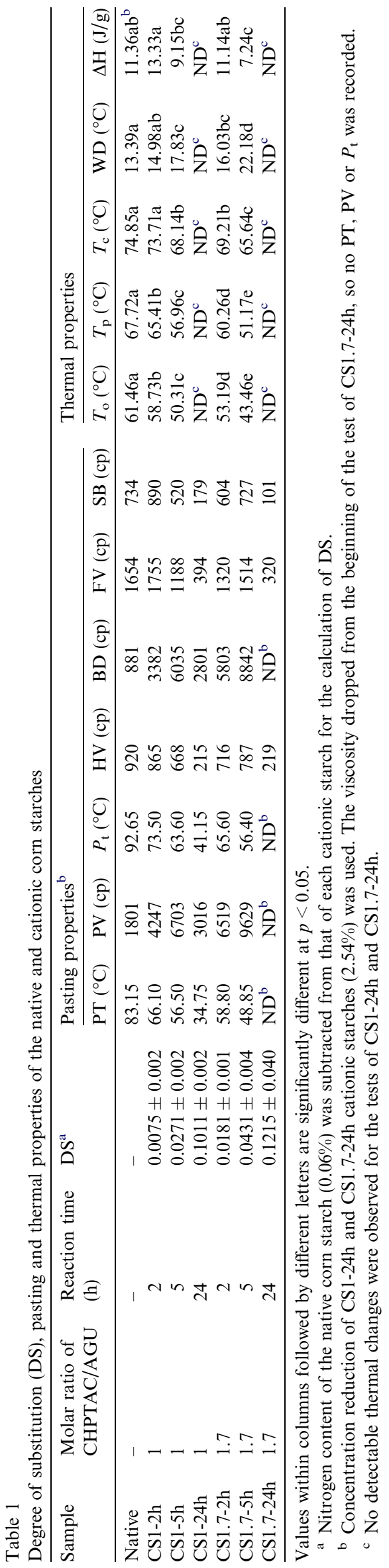

and high PV were observed in cationic starches. The loosened structure of starch granules caused rapid loss of granular integrity, which was also evidenced by the large BD (Kweon et al., 1997; Liu, Corke, \& Ramsden, 1999; Siau et al., 2004). In contrasts with PT, $P_{\mathrm{t}}, \mathrm{PV}$ and BD, SB correlated poorly with DS due to a wide rage of DS (0.0075-0.0431) and few samples (four samples only) was analysized. As reaction time prolonged, SB of samples with lower amount of CHPTAC (CS1-5h) decreased; while those of samples with higher amount of CHPTAC (CS1.7-5h) increased. More than one factor might be involved in the changes of SB of cationic starches. The steric hindrance and Columbic repulsions between cationic starch molecules prevented their reassociation and caused a decrease of SB (Siau et al., 2004). While, the increase in the amount of released amylose resulting from the weak granular structure of cationic starches (Liu et al., 1999) might cause an increase of SB. It has been reported that cationization promoted complete dispersibility of amylose and amylopectin from the granule (Yook et al., 1994). Therefore, the SB of cationic starches depended on the net effect of molecular repulsion and the amount of amylose released during heating. For samples with high DS (CS1.7-5h), the effect of the increasing release of amylose might overwhelm the effect of molecular repulsion, and resulted in a net increase in SB, compared to that of CS1.7-2h. In addition, the homogeneity of starch gel after gelatinization might also have influence on the SB. Granules of cationic starch with high DS burst more easily and completely, thus a homogeneous starch solution, in which the starch molecules and granular fragments rearranged to form a more organized gel structure, was obtained. The FV of the cationic starches was highly correlated with the HV, therefore the highly positive correlation between FV and SB was also observed.

For the sample reacted for $24 \mathrm{~h}$ with high amount of CHPTAC (CS1.7-24h in Table 1), PT, PV and $P_{\mathrm{t}}$ could not be measured due to a sharp drop of viscosity at the beginning of the test. This indicated a rapid water uptake followed by a quick granular burst at the beginning of the test because of weak granular integrity of the cationic starch.

\subsubsection{Thermal properties}

The single narrow gelatinization endotherm in the plot of heat flow versus temperature recorded by DSC is ascribed to the water-mediated melting of starch crystallites, initiated by the stripping of starch chains in the swollen amorphous regions (Aggarwal \& Dollimore, 1998). For CS1-24h and CS1.7-24h, virtually no endothermic heat flow was found in the MDSC tests. This was probably due to overall gelatinization during the 6-h equilibration before the MDSC test. Correlations between DS and the thermal properties were also observed among samples with $\mathrm{DS} \leqq 0.0431$. After cationization, the transition temperatures $\left(T_{\mathrm{o}}, T_{\mathrm{p}}\right.$, and $\left.T_{\mathrm{c}}\right)$ and $\Delta \mathrm{H}$ of the cationic starches decreased. Several researches revealed that chemical substitution served to facilitate the melting of amylopectin 
Table 2

Correlations between DS, pasting and thermal properties of the cationic corn starches (CS1-2h, CS1-5h, CS1.7-2h, and CS1.7-5h) ${ }^{\mathrm{a}}$

\begin{tabular}{|c|c|c|c|c|c|c|c|c|c|c|c|c|}
\hline & $\mathrm{DS}$ & PT & $\mathrm{PV}$ & $P_{\mathrm{t}}$ & $\mathrm{HV}$ & $\mathrm{BD}$ & $\mathrm{FV}$ & $\mathrm{SB}$ & $T_{\mathrm{o}}$ & $T_{\mathrm{p}}$ & $T_{\mathrm{c}}$ & WD \\
\hline PT & $-0.989^{*}$ & & & & & & & & & & & \\
\hline PV & $0.977^{*}$ & $-0.993^{* *}$ & & & & & & & & & & \\
\hline$P_{\mathrm{t}}$ & $-0.983^{*}$ & $0.999^{* *}$ & $-0.990^{* *}$ & & & & & & & & & \\
\hline $\mathrm{HV}$ & -0.320 & 0.385 & -0.300 & 0.420 & & & & & & & & \\
\hline BD & $0.978^{*}$ & $-0.995^{* *}$ & $0.999^{* *}$ & $-0.994^{* *}$ & -0.335 & & & & & & & \\
\hline $\mathrm{FV}$ & -0.346 & 0.412 & -0.328 & 0.447 & $0.999^{* *}$ & -0.363 & & & & & & \\
\hline SB & -0.359 & 0.426 & -0.344 & 0.461 & $0.999^{* *}$ & -0.378 & $1.000^{* *}$ & & & & & \\
\hline$T_{\mathrm{o}}$ & $-0.997^{* *}$ & $0.998^{* *}$ & $-0.988^{*}$ & $0.994^{* *}$ & 0.352 & $-0.990^{*}$ & 0.379 & 0.393 & & & & \\
\hline$T_{\mathrm{p}}$ & $-0.997^{* *}$ & $0.995^{* *}$ & $-0.980^{*}$ & $0.992^{* *}$ & 0.384 & $-0.983^{*}$ & 0.410 & 0.424 & $0.999^{* *}$ & & & \\
\hline$T_{\mathrm{c}}$ & $-0.956^{*}$ & $0.983^{*}$ & $-0.962^{*}$ & $0.990^{*}$ & 0.546 & $-0.972^{*}$ & 0.570 & 0.583 & $0.972^{*}$ & $0.975^{*}$ & & \\
\hline WD & $0.980^{*}$ & $-0.952^{*}$ & $0.955^{*}$ & $-0.937^{*}$ & -0.126 & 0.948 & -0.153 & -0.167 & $-0.969^{*}$ & $-0.963^{*}$ & -0.883 & \\
\hline$\Delta \mathrm{H}$ & $-0.989^{*}$ & $0.982^{*}$ & $-0.954^{*}$ & 0.980 & 0.451 & $-0.948^{*}$ & 0.474 & 0.487 & $0.988^{*}$ & $0.994^{* *}$ & $0.973^{*}$ & -0.942 \\
\hline
\end{tabular}

a Superscripts with the label of $*$ or $* *$ indicated that the correlation was significant at $p<0.05$ or $p<0.01$, respectively.

crystallities (Biliaderis, Maurice, \& Vose, 1980; Biliaderis, 1982). The cationic groups introduced into the starch granule inhibited the inter-chain interaction, and resulted in a loosened structure that consequently became more hydrophilic than that of native starch (Siau et al., 2004). The increase in hydration of starch molecules in the amorphous region provided more water for the crystal melting during heating, thus resulted in a lower transition temperature and a smaller gelatinization enthalpy than that of native starch (Kweon et al., 1997). The WD of the cationic starches was larger than that of native starch and indicated a more diverse, irregular arrangement of starch molecules after the cationization.

\subsection{Effect of cationization on the granular morphology of starches}

\subsubsection{Scanning electron microscopy}

Fig. 1 shows the scanning electron micrographs of the native and cationic starches. Native corn starch (Fig. 1a) was composed of granules with irregular shapes and vari- ous sizes. The surface morphology of low DS cationic starches, CS1-2h and CS1.7-2h (Fig. 1b and e), was similar with that of native corn starch, while more evident pores were observed on some granular surface of CS1-5h and CS1.7-5h (Fig. 1c and f), and some inward concaves were also found in CS1.7-5h. The granules of CS1-24h (Fig. 1d) contained inward concaves, partially disintegrated granules, as well as small fragments. The granules of CS1.7-24h (Fig. 1g) displayed the highest extent of granular fragmentation, and some large granules had especially deep holes. The inward concaves on the granular surface of starch were also reported by Xie, Liu, and Cui (2006) on starch citrates. The cause of the inward concaves might be resulted from the swelling of starch granules during the cationization process, followed by the collapse during cooling after termination of the reaction. The collapsed region is believed to be less organized than the rest of the granule, and is the region where cationization mainly occurred. The increased superficial porosity might facilitate the diffusion of cationizing agent into the inner region, and contribute to the alteration of granular morphology.
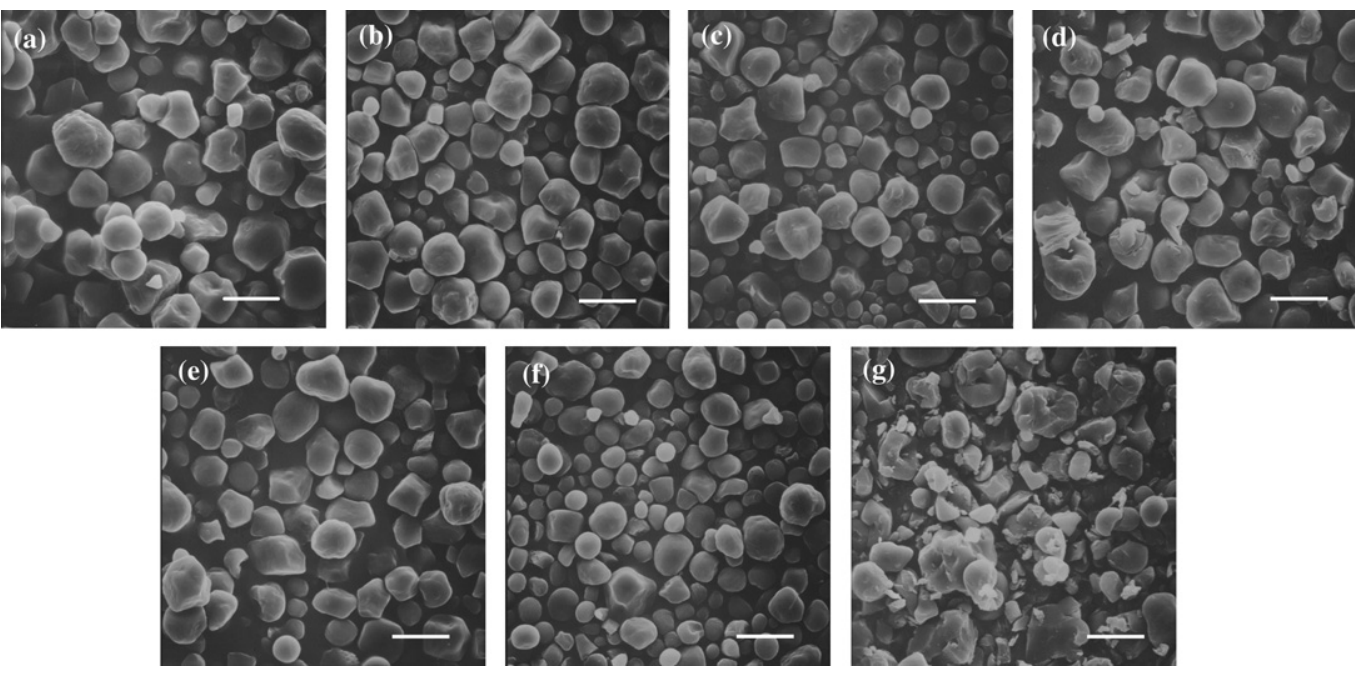

Fig. 1. Scanning electron microscopy of the native (a) and cationic corn starches CS1-2h (b), CS1-5h (c), CS1-24h (d), CS1.7-2h (e), CS1.7-5h (f), and CS1.7-24h (g). Length of scale bar: $15 \mu \mathrm{m}$. 


\subsubsection{Confocal laser scanning microscopy}

Due to the heterogeneity of the internal structure of native corn starch, the diffusion of dyes toward starch granules is a kind of preferential dying, in which certain regions in the granules are more accessible to the dye molecules. It is known that the molecular arrangement around the central helium in a starch granule is less organized than that around the outer area (Buleon, Colonna, Planchot, \& Ball, 1998; Chung \& Lai, 2006). Chung and Lai (2006) showed that the methanolic-APTS solution diffused into the outer layer and passed through the channels into the central cavity, and then migrated from the central cavity toward the internal part of the granule. The regions between granular surface and matrix surrounding interior cavity and channels were the last areas being dyed. In order to monitor the diffusion pathway of the cationic starches, starch granules of CS1.7-5h were dyed against APTS-methanol for different dying times (Fig. 2). At the first $15 \mathrm{~h}$, most of the granules had only the outermost layer dyed while dyed channels and central helium were observed in few of them (Fig. 2a). As time extended to $24 \mathrm{~h}$, the dye spread from the outermost layer and central helium to the interior space (Fig. 2b). After being dyed for $40 \mathrm{~h}$, the interior space was dyed completely, while the internal cavity was enlarged and evident (Fig. 2c) (Velde, Riel, \& Tromp, 2002). These results convinced that the pathway of APTS-methanol toward CS1.7-5h is the same as that toward the native starch. The CLSM observations provided the evidences that the basic internal structure of the cationic starch was not significantly altered during the cationization.

Fig. 3 is the CLSM images of the native and cationic starches dyed against APTS-methanol for $15 \mathrm{~h}$. The area with higher intensity represented the higher amount of reducing ends, which could form covalent bonds with APTS (Chung \& Lai, 2006). In contrast with the native corn starch that had high intensity of dye around the channels and the central region (Fig. 3a), the cationic starches showed high intensity of dye at the outermost layer (Fig. 3b-g). By lowering the integrity of the granular surface of cationic starches, there might be more reducing ends exposed from the granular surface, which then became more vulnerable to the dye. When comparing the cationic starches reacted for 2 and $5 \mathrm{~h}$, the intensity and diffusion areas of dye increased with the increase in DS. This could be an evidence for the loosened arrangement of starch molecules within the starch granules resulting from the introduction of cationic groups. The images of CS1-24h (Fig. 3d) and CS1.7-24h (Fig. 3g) containing swollen granules with completely dyed interior space and large central hollow suggested significant changes in the granular structure. As the results from the determinations of pasting
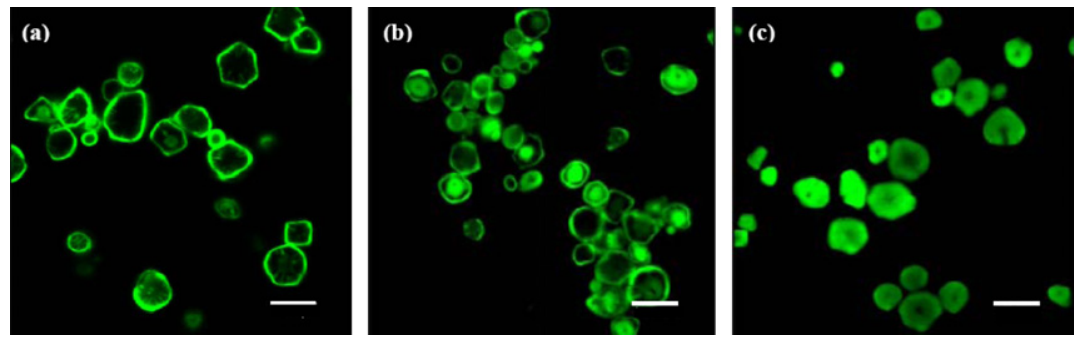

Fig. 2. CLSM optical sections of CS1.7-5h, stained with APTS in methanol for $15 \mathrm{~h}$ (a), $24 \mathrm{~h}$ (b) and $40 \mathrm{~h}$ (c). Length of scale bar: $20 \mu \mathrm{m}$.
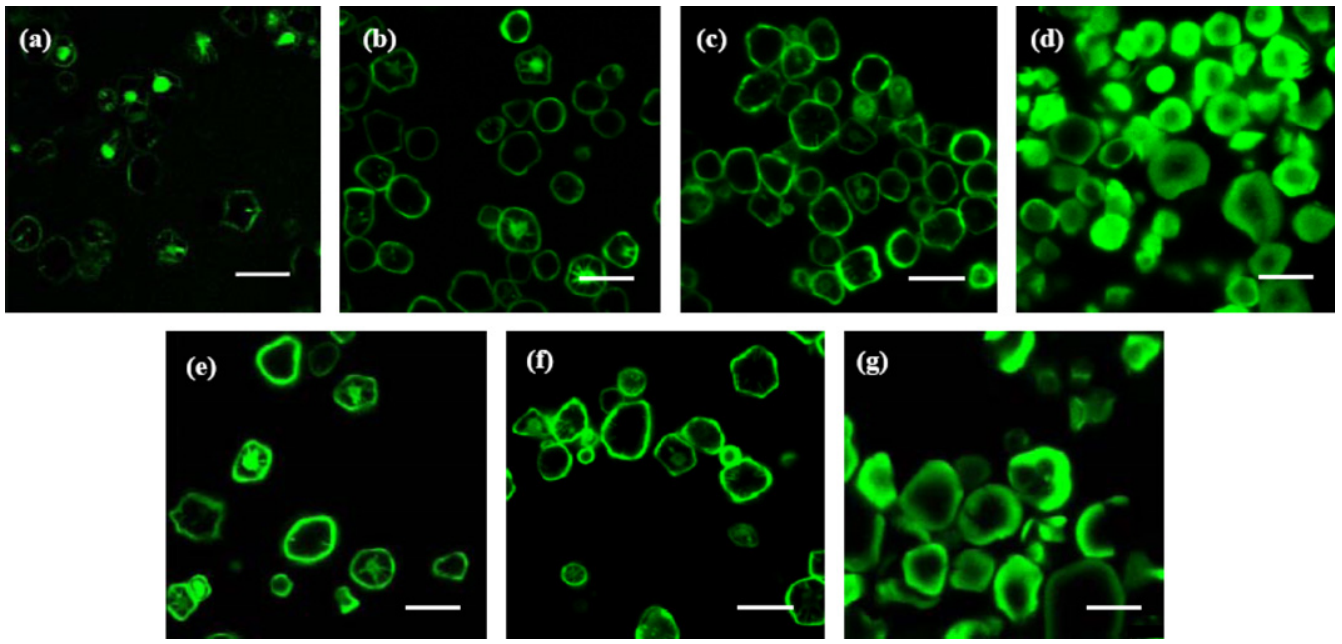

Fig. 3. CLSM optical sections of the native (a) and cationic corn starches CS1-2h (b), CS1-5h (c), CS1-24h (d), CS1.7-2h (e), CS1.7-5h (f), CS1.7-24h (g) stained with APTS in methanol for $15 \mathrm{~h}$. Length of scale bar: $20 \mu \mathrm{m}$. 
and thermal properties, the introduction of cationic groups interrupted the chain-chain interactions between starch molecules. This facilitated the gelatinization of starch granules, and thus resulted in loss of crystallinity and therefore loss of hydrophobic regions (Velde et al., 2002), as evidenced by CLSM images of the thoroughly dyed cationic starches. As the swollen starch granules permitted direct and rapid diffusion of solvent and dye, the expelling of free amorphous components by the invading alcohol was also proceeded at the same time. This phenomenon, called the 'amorphous excretion' by Polaczek, Starzyk, and Tomasik (1999), contributed to the further enlargement of the central black hollow in the swollen starch granules.

\subsubsection{Polarized optical microscopy and bright field microscopy}

Fig. 4 shows the images of polarized optical microscopy (POM) and bright field microscopy (BFM) of the native and cationic starches with or without iodine staining. Like the native starch, cationic starches, except for CS1.7-24h (Fig. 4s), had clear 'Maltese cross' under POM, though the brightness gradually decreased with the increase in DS. This corresponded to the hypothesis that cationic substitution mainly occurred in the amorphous region of starch granules (Yook et al., 1994). The POM image of CS1.7-24h (Fig. 4s) showed a low brightness and loss of birefringence especially at the central region of the granules. The diminished birefringence indicated a reduced bias of the amylopectin molecules toward a radial orientation in the granule. Thus the altered amylopectin structure appeared to be related to the altered molecular orientation (Evans, McNish, \& Thompson, 2003), which was probably caused by the cationizing agent entering the crystalline regions.

The native corn starch reacted with iodine to form a deep, bluish-purple color under BFM (Fig. 4b). The iodine-stained color of the cationic starches changed with the increase of DS. CS1-2h, CS1-5h and CS1.7-2h (Fig. 4e, h and $\mathrm{n}$ ) had slightly swollen granules with a purple color close to that of the native corn starch, while the color of CS1.7-5h (Fig. 4q) turned to red-brown. CS1$24 \mathrm{~h}$ (Fig. 4k) displayed un-uniform colors among granules, of which the swollen or even burst ones showed a much lighter color. The central hallow regions found in some granules were similarly displayed by CLSM (Fig. 3), indicating a phenomenon similar to 'amorphous excretion' might have taken place in this sample during iodine staining. The granules of CS1.7-24h (Fig. 4t) were highly swollen or even burst, with a very light blue color. It has been well known that amylose has strong affinity for iodine with which it forms a deep blue complex, while amylopectin has little affinity for iodine and gives a purple-reddish coloration (Sitohy \& Ramadan, 2001). The color of the amylose-iodine complex also changed depending on the degree of polymerization (DP) of the amylose helix ranging from brown (DP 21-24) to blue (DP > 47) (John, Schmidt, \& Kneifel, 1983). Studies on chemically substituted starches revealed that the substitution might lower the stability of the iodine-amylose complex due to the conformational destabilization of the helical regions (Ogawa et al., 1999). In addition, shortening the effective amylose chain length available for the helical superstructure might result in a weaker iodine color and lower blue value (Breitinger, 2003). Therefore, the changes in color of the iodine-stained cationic starches from blue to purple to red-brown, alone with the increase in DS from 0.0075 to 0.0431 , might be resulted from the cationic substitution that reduced the stability of iodine-amylose complex or the chain length of the iodine-accessible amylose. The much more lightly blue color of CS1.7-24h (Fig. 4t) suggested the presence of relatively small amount of amylose available for iodine complexation. This might be resulted from the significant breakdown of the structural integrity and the leaching out of starch molecules during the cationizing reaction, together with the dilution effect of granular swelling during the iodine staining.

The iodine-stained native and cationic starches showed various colors and morphologies under POM. The native corn starch had a clear 'Maltese cross' and a blue color under POM (Fig. 4c), while CS1-2h, CS1-5h, CS1.7-2h and CS1.7-5h (Fig. 4f, i, o and r) showed more irregular granules and a color between purple and pink. CS1-24h (Fig. 41) and CS1.7-24h (Fig. 4u) under POM had diminished 'Maltese cross.' The granular starch appeared pale blue, while the highly swollen or burst starch granules appeared light brown. The color of iodine-stained starch under POM had been related to the molecular organization in granules of starch from different genotypes (Evans et al., 2003). After iodine staining, the granules with both amylose and amylopectin oriented radically appear blue under POM, while those with only radically oriented amylopectin appear pink. Therefore, the changes in color of the cationic starches under POM from purple to pink with the increase in DS up to 0.0431 might indicate the alteration in conformation and molecular arrangement of amylose, instead of amylopectin, after being substituted with cationic groups. Cationization facilitated water penetration in starch granules. When being stained with aqueous iodine solution, the granules of cationic starches with high DS (CS1.7-24h and CS1-24h) absorbed water quickly and resulted in a rapid decline in crystallinity, thus a loss of 'Maltese cross' occurred.

The results of granular morphology of the cationic starches, investigated by SEM, BFM, POM as well as CLSM, correlated reasonably with the results of physicochemical properties investigated by RVA and MDSC. All results revealed gradual reduction of the granular integrity of the cationic starches with DS up to 0.0431, while significant alteration of granular structure took place in cationic starch with high DS (0.1215).

\subsubsection{Wide angle $X$-ray diffraction pattern}

Fig. 5 displays the X-ray diffractograms of native and cationic starches. Native corn starch exhibited a character- 

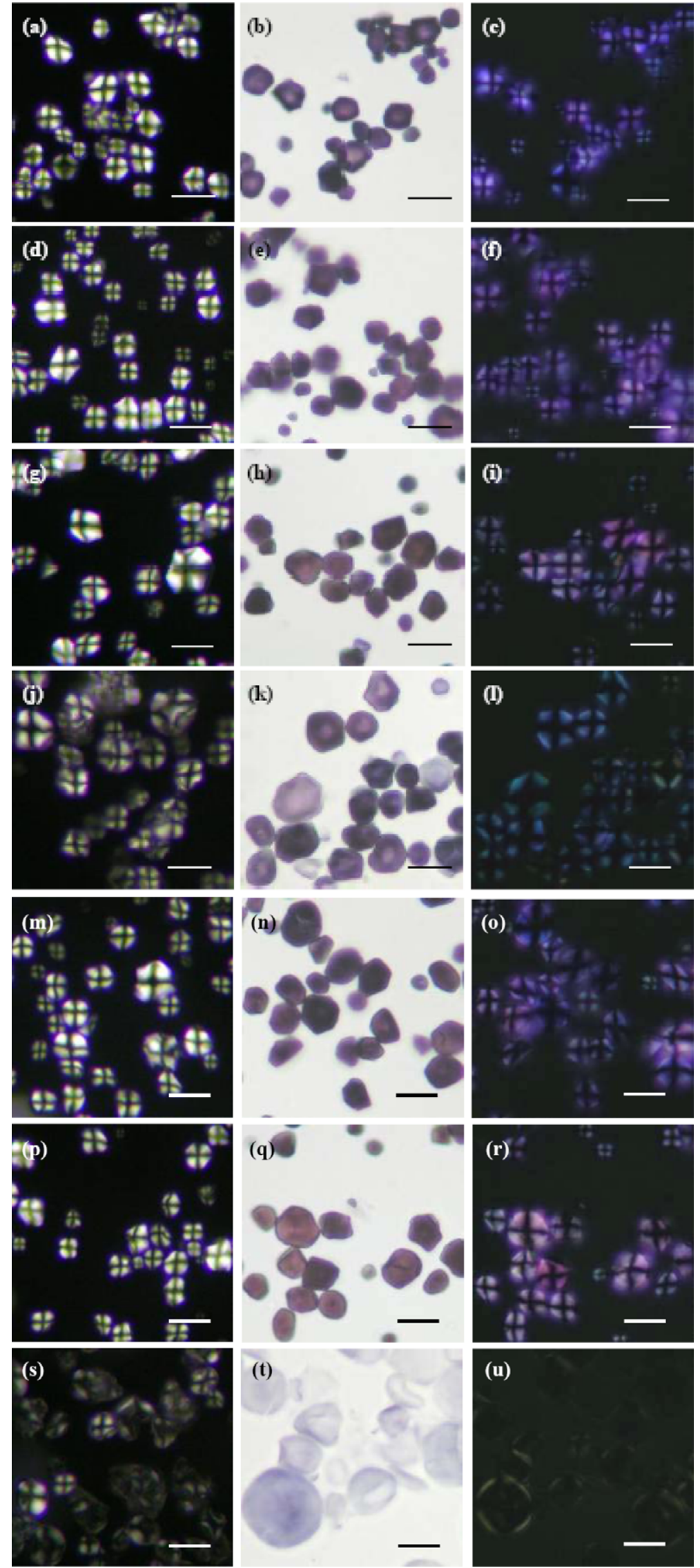

Fig. 4. POM (left and right), BFM (middle) images of the native (a-c) and cationic corn starches CS1-2h (d-f), CS1-5h (g-i), CS1-24h (j-1), CS1.7-2h $(\mathrm{m}-\mathrm{o}), \mathrm{CS} 1.7-5 \mathrm{~h}(\mathrm{p}-\mathrm{r})$, and CS1.7-24h (s-u) stained with (middle and right) or without (left) iodine. Length of scale bar: $20 \mu \mathrm{m}$. 


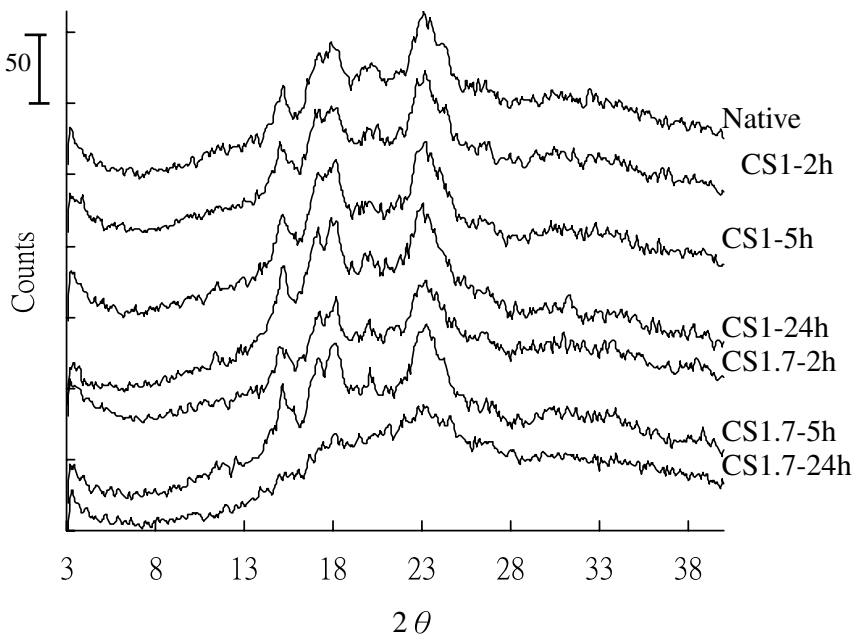

Fig. 5. Wide angle X-ray diffraction patterns of the native and cationic corn starches.

istic A-type X-ray diffraction pattern with evident intensity lines at the mean interplanar spacing of $0.547,0.485$, and $0.361 \mathrm{~nm}$. Cationic starches, except for CS1.7-24h, had diffraction patterns similar to that of native starch. This result again supported the hypothesis that the substitution of cationic groups had little effects on the crystalline structure, but rather concentrated primarily in the amorphous regions of starch granules (Aggarwal \& Dollimore, 1998; Segura \& Sira, 2003). In contrast, the diffractogram of CS1.7-24h showed the complete lack of reflection bands, a typical diffraction pattern of amorphous samples, and suggested that most of the crystalline region of this sample was disrupted. While cationic substitution might have changed the crystallinity to some degree, the thorough collapse of crystalline structure of this sample might be due to high amount of water absorption during the equilibration under high relative humidity prior to the measurements. After extensive cationization, the reduction in the integrity of granules resulted in the increase in water uptake and migration rate inside the granules. The similar effects could also be seen from the POM of the iodine-stained starch (Fig. $4 \mathrm{u}$ ) and from the RVA and MDSC results of this sample (Table 1).

\section{Conclusions}

The cationic starches in the granular form with DS ranging from 0.0075 to 0.1215 were derived from aqueous phase cationization without the addition of swelling-inhibiting salts. For the cationic starches with DS $\leqq 0.0431$, of which substitution mainly occurred in amorphous regions of the granules, the pasting and thermal properties, as well as the structural morphology changed gradually and correspondingly with the increase of DS. For the cationic starch with DS as high as 0.1215 the cationizing agent might enter the crystalline regions because the significant changes in physicochemical properties and evident reduction in granular integrity had been observed.

\section{Acknowledgements}

Authors want to thank Dr. Ming-Kuang Wang for assistance in the measurements of X-ray diffractometry. This work was supported by the Grant NSC 94-2815-C002-128-B from the National Science Council, Taipei, Taiwan.

\section{References}

AACC (2000). Approved methods of the AACC, 10th ed. Method 46-11A, 08-01, 30-20 and 46-12. St. Paul, Minnesota: American Association of Cereal Chemists, Inc.

Aggarwal, P., \& Dollimore, D. (1998). The effect of chemical modification on starch studied using thermal analysis. Thermochimica Acta, 324, $1-8$.

Biliaderis, C. G. (1982). Physical characteristics, enzymatic digestibility and structure of chemically-modified smooth pea and waxy maize starch. Journal of Agricultural and Food Chemistry, 30, 925-930.

Biliaderis, C. G., Maurice, T. J., \& Vose, J. R. (1980). Starch gelatinization phenomena studied by differential scanning calorimetry. Journal of Food Science, 45, 1669

Breitinger, H. G. (2003). Synthesis and characterization of 2,3-di-Oalkylated amyloses: hydrophobic substitution destabilizes helical conformation. Biopolymers, 69, 301-310.

Buleon, A., Colonna, P., Planchot, V., \& Ball, S. (1998). Starch granules: structure and biosynthesis. International Journal of Biological Macromolecules, 23, 85-112.

Chung, Y. L., \& Lai, H. M. (2006). Molecular and granular characteristics of corn starch modified by HCl-methanol at different temperatures. Carbohydrate Polymers, 63, 527-534.

Evans, A., McNish, N., \& Thompson, D. B. (2003). Polarization colors of lightly iodine-stained maize starch for amylose-extender and related genotypes in the W64A inbred line. Starch/Stärke, 55, 250-257.

Gimmler, N., \& Meuser, F. (1994). Influence of extrusion cooking conditions on the efficiency of the cationisation and carboxymethylation of potato starch granules. Starch/Stärke, 46, 268-276.

Heinze, T., Haack, V., \& Rensing, S. (2004). Starch derivatives of high degree of functionalization. 7. Preparation of cationic 2-hydroxypropyltrimethylammonium chloride starch. Starch/Stärke, 56, 288-296.

Hellwig, G., Bischoff, D., \& Rubo, A. (1992). Production of cationic starch ethers using an improved dry process. Starch/Stärke, 44, 69-74.

Huber, K. C., \& BeMiller, J. N. (2003). Location of sites of reaction within starch granules. Cereal Chemistry, 78(2), 173-180.

Jensen, B. M., \& Winters, J. C. (1986). Cationic potato starch proven effective as wet-end additives. Pulp \& paper, 60(4), 83-85.

John, M., Schmidt, J., \& Kneifel, H. (1983). Iodine-maltosaccharide complexes: relation between chain-length and colour. Carbohydrate Research, 119, 254-259.

Krentz, D. O., Lohmann, C., Schwarz, S., Bratskaya, S., Liebert, T., Laube, J., et al. (2006). Properties and flocculation efficiency of highly cationized starch derivatives. Starch/Starke, 58, 161-169.

Kweon, M. R., Hanover, E., Sosulski, F. W., \& Han, H. S. (1997). Effect of aqueous ethanol cationization on functional properties of normal and waxy starches. Starch/Starke, 49, 202-207.

Liu, H., Corke, H., \& Ramsden, L. (1999). Functional properties and enzymatic digestibility of cationic and cross-linked cationic $a e, w x$, and normal maze starch. Journal of Agricultural and Food Chemistry, 47, 2523-2528.

Nachtergaele, W. (1989). The benefits of cationic starches for the paper industry. Starch/Starke, 41, 27-31.

Ogawa, K., Hirai, I., Shimasaki, C., Yoshimura, T., Ono, S., Rengakuji, S., et al. (1999). Simple determination method of degree of substitution for starch acetate. Bulletin of the Chemical Society of Japan, 72, 2785-2790. 
Polaczek, E., Starzyk, F., \& Tomasik, P. (1999). Starch-alcohol complexes. Carbohydrate Polymers, 39, 37-42.

Radosta, S., Vorwerg, W., Ebert, A., Begli, A. H., Grülc, D., \& Wastyn, M. (2004). Properties of low-substituted cationic starch derivatives prepared by different derivatisation processes. Starch/Starke, 56(7), $277-287$.

Sakai-Kato, K., Kato, M., Nakajima, T., Toyo'oka, T., Imai, K., \& Utsunomiya-Tate, N. (2006). Cationic starch derivatives as dynamic coating additives for protein analysis in capillary electrophoresis. Journal of Chromatography A, 1111, 127-132.

Segura, M. E. M., \& Sira, E. E. P. (2003). Characterization of native and modified cassava starches by scanning electron microscopy and X-ray diffraction techniques. Cereal Chemistry, 48(2), 173-180.

Siau, C. L., Karim, A. A., Norziah, M. H., \& Wan Rosli, W. D. (2004). Effects of cationization on DSC thermal profiles, pasting and emulsifying properties of sago starch. Journal of the Science of Food and Agriculture, 84, 1722-1730.

Siemion, P., Kapusniak, J., \& Koziol, J. (2005). Solid-state thermal reactions of starch with semicarbazide hydrochloride. Cationic starch of a new generation. Carbohydrate Polymers, 62, 182-186.

Sitohy, M. Z., \& Ramadan, M. F. (2001). Granular properties of different starch phosphate monoesters. Starch/Stärke, 53, 27-34.
Solarek, D. B. (1986). Cationic starches. In O. B. Wurzburg (Ed.), Modified starches: Properties and uses (pp. 113-128). Boca Raton, FL: CRC Press.

Valle, G. D., Colonna, P., \& Tayeb, J. (1991). Use of a twin-screw extruder as a chemical reactor for starch cationization. Starch/Starke, 43, 300-307.

Velde, F. V. D., Riel, J. V., \& Tromp, R. H. (2002). Visualisation of starch granule morphologies using confocal scanning laser microscopy (CSLM). Journal of the Science of Food and Agriculture, 82(13), 1528-1536.

Vihervaara, T., Bruun, H. H., Backman, R., \& Paakkanen, M. (1990). The effect of different methods of cationization on the starch granule and its gelatinisation product. Starch/Starke, 42, 64-68.

Wurzburg, O. B. (1986). Forty years of industrial starch research. Cereal Food World, 31, 897-903.

Xie, X., Liu, Q., \& Cui, S. W. (2006). Studies on the granular structure of resistant starch (type 4) from normal, high amylose and waxy corn starch citrates. Food Research International, 39, 332-341.

Yook, C., Sosulski, F., \& Bhirud, P. R. (1994). Effects of cationization on functional properties of pea and corn starch. Starch/Starke, 46, 393-399. 